\title{
Structural and Dynamical Analysis of 0.1 pc Cores and Filaments in the 30 Doradus-10 Giant Molecular Cloud
}

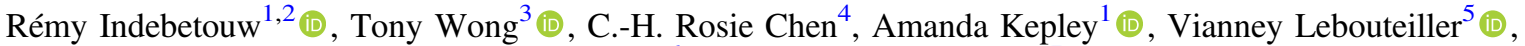 \\ Suzanne Madden ${ }^{6}$ (D), and Joana M. Oliveira ${ }^{7}$ (D) \\ ${ }^{1}$ National Radio Astronomy Observatory, 520 Edgemont Road, Charlottesville, VA 22903, USA; rindebet@nrao.edu, akepley@nrao.edu \\ ${ }^{2}$ Department of Astronomy, University of Virginia, P.O. Box 3818, Charlottesville, VA 22903-0818, USA; remy@virginia.edu \\ ${ }^{3}$ Department of Astronomy, University of Illinois, 1002 W. Green St., Urbana, IL 61801, USA; wongt@illinois.edu \\ ${ }^{4}$ Max Planck Institute for Radio Astronomy, 53121 Bonn, Germany \\ ${ }^{5}$ AIM, CEA, CNRS, Université Paris-Saclay, Université Paris Diderot, Sorbonne Paris Cité, F-91191 Gif-sur-Yvette, France; vianney.lebouteiller@cea.fr \\ ${ }^{6}$ IRFU, CEA, Université Paris-Saclay, F-91191 Gif-sur-Yvette, France; suzanne.madden@cea.fr \\ ${ }^{7}$ School of Physical and Geographical Sciences, Lennard-Jones Laboratories, Keele University, Staffordshire ST5 5BG, UK; j.oliveira@keele.ac.uk \\ Received 2019 August 22; revised 2019 November 22; accepted 2019 November 29; published 2020 January 8
}

\begin{abstract}
High-resolution $(<0.1 \mathrm{pc}$ ) Atacama Large Millimeter/submillimeter Array (ALMA) observations of the 30Dor-10 molecular cloud $15 \mathrm{pc}$ north of R136 are presented. The ${ }^{12} \mathrm{CO} 2-1$ emission morphology contains clumps near the locations of known mid-IR massive protostars, as well as a series of parsec-long filaments oriented almost directly toward R136. There is elevated kinetic energy (line widths at a given size scale) in 30Dor-10 compared to other Large Magellanic Cloud and Galactic star formation regions, consistent with large-scale energy injection to the region. Analysis of the cloud substructures is performed by segmenting emission into disjoint approximately round "cores" using clumpfind, by considering the hierarchical structures defined by isointensity contours using dendrograms, and by segmenting into disjoint long thin "filaments" using Filfinder. Identified filaments have widths $\sim 0.1 \mathrm{pc}$. The inferred balance between gravity and kinematic motions depends on the segmentation method: entire objects identified with clumpfind are consistent with freefall collapse or virial equilibrium with moderate external pressure, whereas many dendrogram-identified parts of hierarchical structures have higher mass surface densities $\Sigma_{\text {LTE }}$ than if gravitational and kinetic energies were in balance. Filaments have line masses that vary widely compared to the critical line mass calculated assuming thermal and nonthermal support. Velocity gradients in the region do not show any strong evidence for accretion of mass along filaments. The upper end of the "core" mass distribution is consistent with a power law with the same slope as the stellar initial mass function.
\end{abstract}

Unified Astronomy Thesaurus concepts: Large Magellanic Cloud (903); Interstellar medium (847); Star formation (1569); Star forming regions (1565); Stellar feedback (1602); Molecular clouds (1072)

\section{Introduction}

One major open question in star formation is how gas is accreted from the clump and cloud scale $(\sim 10 \mathrm{pc})$ onto nascent stars. For many years there has been debate about whether isolated cores $(\sim 0.1 \mathrm{pc})$ form and then collapse into individual stars and multiples, or whether a significant fraction of the eventual stellar mass is accreted from farther away in the cluster gravitational potential well (often referred to as competitive or collaborate accretion). A related question is whether the stellar initial mass function (IMF) is already set by cloud fragmentation into the core mass function, before most of the matter has accreted onto protostars (e.g., Motte et al. 2018, and references therein). Over the past decade, it has become clear that in solar neighborhood clouds, filamentary structures $(\sim 0.1 \mathrm{pc} \times 1 \mathrm{pc})$ are ubiquitous, contain most of the dense cores in such clouds, and likely play a role in transferring matter from the cloud to core scales (e.g., Fernández-López et al. 2014; André 2015).

To develop any universal understanding of star formation, it is important to determine whether the same core and filament structures exist in a wider range of molecular clouds than exist in the solar neighborhood. Distant regions in the Milky Way disk suffer from both significant distance (and thus source luminosity) uncertainty and confusion along the line of sight. The Large Magellanic Cloud (LMC) is not much farther but, being close to face-on, suffers from little distance uncertainty or line-of-sight confusion. The 30Dor-10 molecular cloud (Johansson et al. 1998) is located $15 \mathrm{pc}$ to the NE of the rich star cluster R136 in the LMC. The UV radiation field affecting 30 Dor-10 is $3500 \times$ that of the solar neighborhood (Werner et al. 1978), and the reduced dust abundance at the region's $\sim 1 / 2$ solar metallicity (Russell \& Dopita 1992; Peimbert 2003) makes the interstellar medium more permeable to that radiation (Poglitsch et al. 1995). This makes 30Dor-10 a good laboratory in which to study star formation in the presence of strong external radiative feedback. 30 Doradus is also remarkable compared to typical star formation regions in the Magellanic Clouds or Milky Way, and perhaps signatures of what caused such an intense star formation event are still evident in the structure of the residual molecular gas. Low- and intermediatemass star formation is actively ongoing in 30Dor-10 (Rubio et al. 1998; Walborn et al. 2013; Sabbi et al. 2016), and now with the Atacama Large Millimeter/submillimeter Array (ALMA) we can obtain a detailed picture of the molecular gas down to $0.1 \mathrm{pc}$ scales.

In ALMA Cycle 0 we mapped the 30Dor- 10 cloud in ${ }^{12} \mathrm{CO}$ ${ }^{2-1},{ }^{13} \mathrm{CO} 2-1, \mathrm{C}^{18} \mathrm{O} 2-1$, and $1.3 \mathrm{~mm}$ continuum at a resolution of $\simeq 2$ !" $3 \times 1$ !" $5 \quad$ (2011.0.00471.S, Indebetouw et al. 2013, hereafter Paper I), equaling $=0.56 \mathrm{pc} \times 0.36 \mathrm{pc}$ at the adopted distance of $50 \mathrm{kpc}$ (e.g., de Grijs et al. 2014). We found that resolved parsec-scale structures have $>3 \times$ larger velocity line widths at those scales than other star formation 
regions in the Milky Way and LMC (Indebetouw et al. 2013; Nayak et al. 2016). This can be explained by an external pressure of $P_{e} / k>10^{6} \mathrm{~cm}^{-3} \mathrm{~K}$, either from the bubble and ionized regions around R136 or merely from the weight of the molecular cloud envelope in which the observed clumps are embedded. The slope of the size-line width relation agreed with other regions within uncertainties, and there were no strong trends of clump property with distance from R136, so the question whether star-forming clumps in 30Dor-10 are affected by feedback, or whether the entire region is simply very turbulent, was unresolved. This study extends the previous work to much smaller angular resolution, now resolving the $0.1 \mathrm{pc}$ sized cores that are expected to be actively participating in individual or multiple star formation. The greater spatial dynamic range permits more robust determination of size-line width-mass relations, and a core mass function more directly relevant to star formation can be measured, as described in the following sections.

\section{Observations and Data Reduction}

The 30Dor- 10 cloud was observed with ALMA in ${ }^{12} \mathrm{CO} 2-1$, ${ }^{13} \mathrm{CO} 2-1, \mathrm{C}^{18} \mathrm{O} 2-1,1.3 \mathrm{~mm}$ continuum, the $\mathrm{H} 30 \alpha$ recombination line, and $\mathrm{H}_{2} \mathrm{CO} 3_{0,3}-2_{0,2}, \quad 3_{2,2}-2_{2,1}$, and $3_{2,1}-2_{2,0}$ (218.22219, 218.47563, and $218.76007 \mathrm{GHz}$; formaldehyde data will be presented separately). Most of the cloud was mapped as part of project 2011.0.00471.S (Indebetouw et al. 2013 ) at a resolution of $\simeq 2 ! .3 \times 1 . ! 5=0.56 \mathrm{pc} \times 0.36 \mathrm{pc}$. The brightest parts of the cloud were observed in project 2013.1.00346, targeting the same lines at higher angular $(<0.1 \mathrm{pc})$ and spectral resolution. $\left(\mathrm{C}^{18} \mathrm{O} 2-1\right.$ and the $\mathrm{H}_{2} \mathrm{CO}$ lines were observed at $122 \mathrm{kHz}=168 \mathrm{~m} \mathrm{~s}^{-1}$ resolution, ${ }^{12} \mathrm{CO}$ $2-1$ and ${ }^{13} \mathrm{CO} 2-1$ at $61 \mathrm{kHz} \simeq 80 \mathrm{~m} \mathrm{~s}^{-1}$ resolution.) The observation was executed 7 times between 2015 June 27 and 2015 September 24. The phase calibrator was J0635-7516 $(0.46-0.53 \mathrm{Jy}$ at $230.5 \mathrm{GHz}$ during the time range of observations). J0635-7516 was also used as a bandpass calibrator in all but one execution that used J0538-4405 (1.2 Jy at $230.5 \mathrm{GHz}$ ). The amplitude calibrator was J0519-454 (0.66-1.0 Jy at $230.5 \mathrm{GHz}$ ). Data were calibrated using the ALMA Calibration pipeline version Cycle3R1 included in Common Astronomy Software Applications (CASA; http://casa.nrao.edu) ver. 4.3.1 (McMullin et al. 2007). Calibrated visibilities were subsequently continuum-subtracted in the $u v$ domain and deconvolved using clean and tclean in CASA 4.5, and a prototype version of the auto-multithresh automasking routine now included in CASA (since ver. 5.1.0; Kepley et al. 2020). Visibilities from both projects were included in the deconvolution. The ${ }^{12} \mathrm{CO} 2-1$ and ${ }^{13} \mathrm{CO} 2-1$ data were imaged at $0.4 \mathrm{~km} \mathrm{~s}^{-1}$ resolution with Briggs weighting, robust $=0.5$, and multiscale deconvolution at 0,5 , and 9 times the $0 . " 032$ pixel, achieving a beam of 0 ." $31 \times 0$. ". $22=0.08 \mathrm{pc} \times 0.05 \mathrm{pc}$ and rms noise in line-free channels of $2.3 \mathrm{mJy} \mathrm{beam}^{-1}$. The ${ }^{12} \mathrm{CO}$ 2-1 image was feathered with APEX single-dish data to recover all of the $\sim 25 \%$ of the large-scale emission that was resolved out by the interferometer; the combined image used for subsequent analysis has an rms of $6 \mathrm{mJy} \mathrm{bm}^{-1}$. Comparison of the ALMA and APEX data for ${ }^{13} \mathrm{CO} 2-1$ indicates that the interferometer recovered all emission to within uncertainties, so the ALMA-only image is used for ${ }^{13} \mathrm{CO} 2-1$ analysis. The $\mathrm{C}^{18} \mathrm{O} 2-1$ data were imaged at $0.4 \mathrm{~km} \mathrm{~s}^{-1}$ resolution with natural weighting, achieving a beam of 0 ". $44 \times 0$ "! 28 and $\mathrm{rms}=1.6 \mathrm{mJy}^{\text {beam }^{-1}}$.

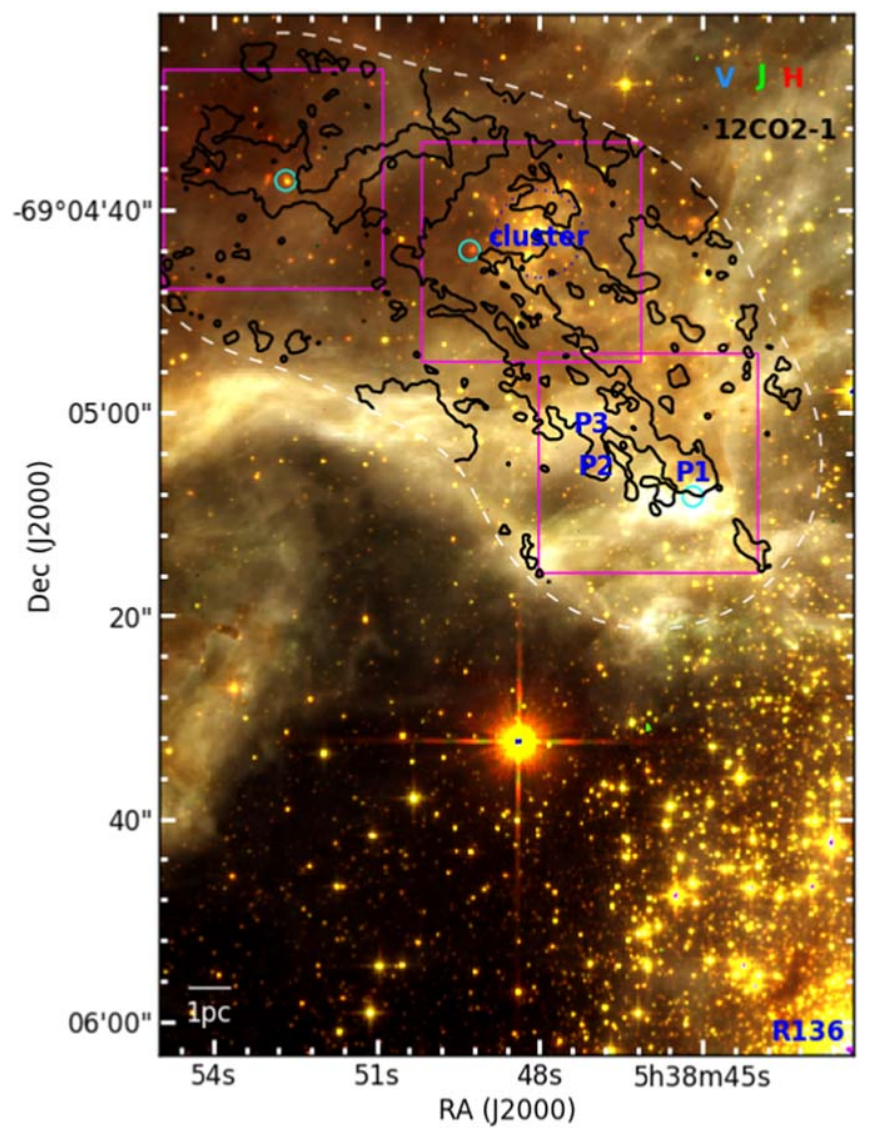

Figure 1. The black contour shows the $3 \sigma^{12} \mathrm{CO} 2-1$ peak intensity 30Dor-10, overlaid on an RGB composite of $H S T$ F160W $\sim H$, F110W $\sim J$, and F658N $(\mathrm{H} \alpha)$. The $\mathrm{CO}$ beam is the small black dot to the left of the " $12 \mathrm{CO} 2-1$ " label. The dominant sources of ionizing radiation are the stars to the southwest, including R136. Pillar structures P1, P2, and P3 and a small cluster in the center of the cloud are labeled in blue, and the three Spitzer-identified massive YSOs in this region are marked in cyan-see text for discussion. Figure 2 shows the CO structure in more detail. Figure 3 shows zoom-ins of the three regions in magenta.

\section{CO Morphology and Associated Star Formation}

Figure 1 shows the extent of ${ }^{12} \mathrm{CO} 2-1$ emission relative to optical and near-IR (NIR) emission imaged with Hubble Space Telescope (HST) for the HTTP project (Sabbi et al. 2013) and the location of features that will be discussed in this section. Figure 2 shows the peak and integrated emission of ${ }^{12} \mathrm{CO} 2-1$ and ${ }^{13} \mathrm{CO} 2-1$, and Figure 3 shows three zoomed-in subregions in F160W $\sim H, \mathrm{~F} 110 \mathrm{~W} \sim J$, and $1 \mathrm{~mm}$ continuum.

Nearest R136 in the southwest, the CO-traced molecular gas is characterized by photodissociated pillars (P1, P2, and P3 in Figure 1), each of which has embedded $1 \mathrm{~mm}$ sources visible in the right panel of Figure 3. Interestingly, the pillar "P1" closest to R136 in projection happens to be the largest sized pillar and contains two very embedded young stellar objects (YSOs), only detected in the millimeter continuum. Just in "front" (SW, i.e., on the R136 side) of the pillar are two NIR-detected, more exposed or evolved stars. This sequence of more embedded sources being farther back from the photodissociating source has been studied in the Milky Way and cited as evidence for triggered star formation by compression of the pillar heads, but causality is challenging to establish (Fukuda et al. 2002; Dale et al. 2015). A $1720 \mathrm{GHz} \mathrm{OH}$ maser was detected at the pillar position (Figure 3, right panel; Brogan et al. 2004), but those 

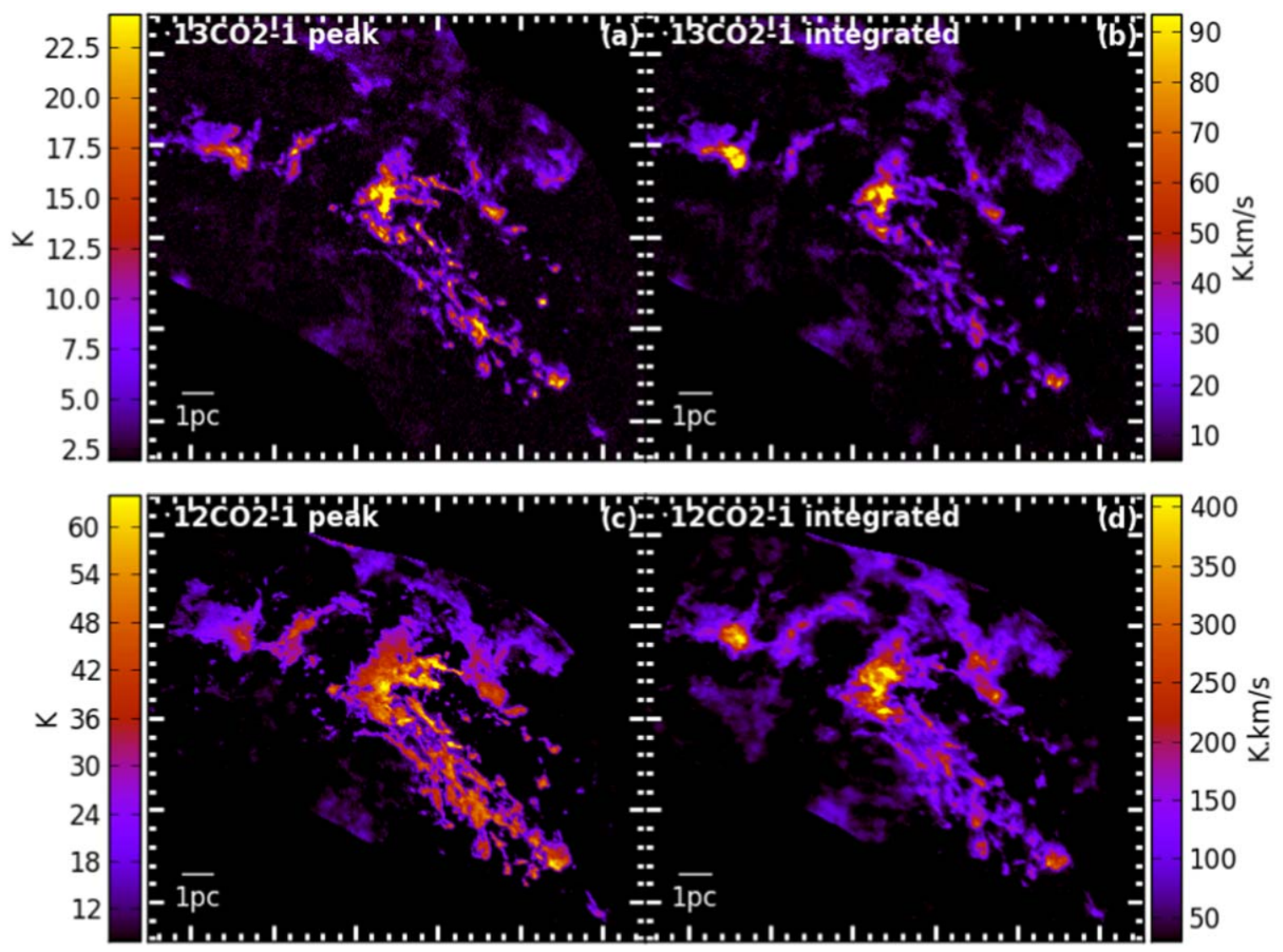

Figure 2. Peak and integrated ${ }^{13} \mathrm{CO} 2-1$ and ${ }^{12} \mathrm{CO} 2-1$ at $0.1 \mathrm{pc}$ resolution in 30Dor-10 (beams are small white dots to the left of each label).

data had insufficient angular resolution to associate the maser with the embedded protostars, or the outer part of the pillar compressed by the $\mathrm{H}$ II region. The $1720 \mathrm{GHz} \mathrm{OH}$ masers are often associated with supernova remnant-molecular cloud interactions, and a similar shock could be being driven into the pillar, but such masers are also associated with massive YSOs (e.g., Gray et al. 1992). Pillar 1 is also associated with one of three "massive YSOs" (MYSOs) identified in this region with Spitzer (Whitney et al. 2008; Gruendl \& Chu 2009; Walborn et al. 2013). However, even with IRAC at $\leqslant 8 \mu \mathrm{m}$, Spitzer's resolution is nearly a half parsec, so its not possible to distinguish a small cluster from an individual massive protostar. With HST's resolution of $\sim 15,000$ au, resolved red NIR sources at the positions of the red Spitzer sources can be much more convincingly called single or multiple MYSOs, and now with ALMA the small-scale structure of their associated molecular gas is resolved. The Pillar 1 MYSO is clearly a small group, including the more embedded millimeter continuum sources and the more exposed NIR sources in the bright photoionized rim.

Slightly farther away from R136 in projection $(\sim 3 \mathrm{pc})$ are two other pillars, $\mathrm{P} 2$ and $\mathrm{P} 3$, which each contain a YSO visible in the NIR. Both embedded sources are slightly extended in NIR and $1 \mathrm{~mm}$ continuum.

The main part of the region, between the southwest pillars and the central cluster and clumps, is characterized by multiple narrow filaments pointing approximately at the central stellar cluster R136. Photoionization can erode clouds, leaving shadowed structures and pillars pointing toward the radiation source (Gritschneder et al. 2010), but it is also possible that this structure is instead related to the formation of 30 Doradus:
Rahner et al. (2018) model the region as an older burst of star formation, followed by recollapse and formation of R136, at high molecular cloud mass and density. Although their model is only 1D, repeated feedback-driven recollapse and nonspherical re-expansion could naturally lead to dense radial structures.

In the center of the mapped region is a small $(\sim 1.5 \mathrm{pc}$ diameter; see Figure 1) intermediate-mass cluster associated with diffuse $\mathrm{H} \alpha$ emission and at least one very massive star (O3-6; Walborn \& Blades 1997). Remnant filamentary COtraced molecular gas is coincident with the eastern side of the cluster, likely in front of the cluster, since the CO filaments correspond to shadows in the diffuse $\mathrm{H} \alpha$. These filaments may be under compression by the expanding ionized region, but there is no evidence in millimeter continuum or NIR of embedded protostars. Just east of the cluster is the brightest $\mathrm{CO}$ clump in our data, now resolved into a southern core with an embedded NIR source and a northern "hub" with filaments leading into it (although there are no clear kinematic signatures of accretion along those spokes). The northern source has an extended $1 \mathrm{~mm}$ source, not detected in the NIR, and is likely the young MYSO that dominates the mid-IR emission from the region (Spitzer cannot resolve these two MYSOs). That more embedded northern source is associated with an $\mathrm{H}_{2} \mathrm{O}$ maser (Imai et al. 2013), thought to be collisionally pumped, and most commonly associated with massive YSO outflows (Elitzur et al. 1989; Walsh et al. 2011). The "hub-and-spoke" morphology of the CO emission is also commonly seen in Galactic MYSOs forming in molecular clouds. Thus, although the source is suggestively located on the edge of the small $\mathrm{H}$ II region, it is difficult to unambiguously claim that its formation was triggered by that $\mathrm{H}$ II region. On the western side of the small 

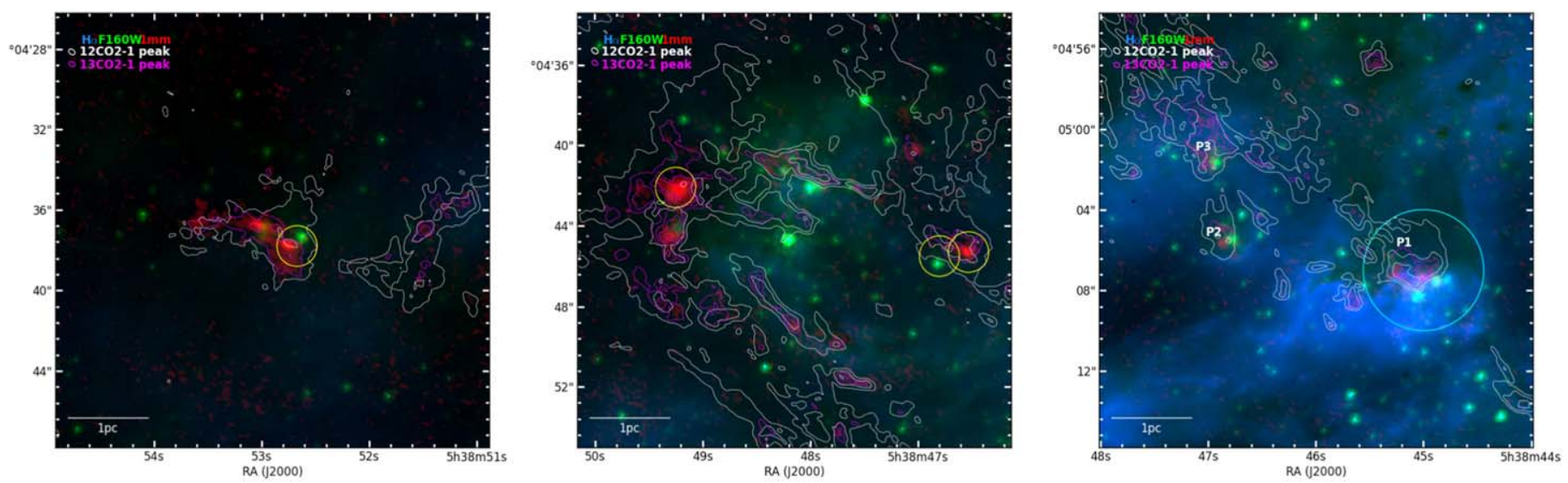

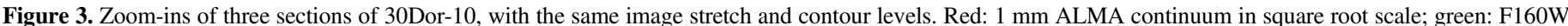

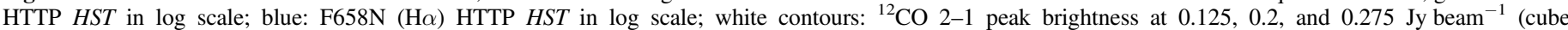

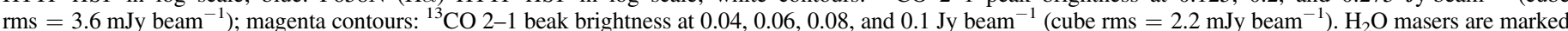

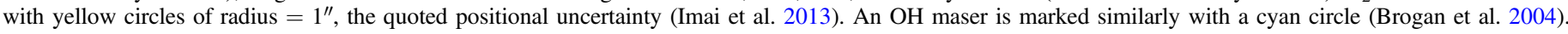

cluster is another $\mathrm{CO}$ clump coincident with two detected $\mathrm{H}_{2} \mathrm{O}$ masers. One is at the position of a $1 \mathrm{~mm}$ continuum source; the other might coincide with an NIR-detected star.

On the eastern side of our mapped area, farthest from R136, is another very massive clump, with at least one deeply embedded massive protostar detected in $1 \mathrm{~mm}$ continuum. The kinematic structure is complex and suggestive of multiple outflows. It is not clear whether the NIR source is an illuminated outflow cavity from the massive protostar(s) or an unassociated source.

\section{CO 2-1 Structure Analysis}

\subsection{Methods}

Molecular clouds have complex hierarchical structure on a large range of spatial scales, but there is insight to be gained by segmenting the emission and analyzing it as discrete structures. The most common technique has been segmentation of position-position-velocity cubes into approximately round entities a few times the spatial resolution-these are typically called clumps or cores, for $\sim$ parsec-sized and $\sim 0.1 \mathrm{pc}$ sized entities, respectively. The segmentation process begins by finding local maxima, with two critical parameters: $\delta I$, the intensity difference between a local maximum and highest connected saddle point, and $I_{\min }$, the minimum intensity to consider. Neighboring pixels are assigned to each local maxima to define a set of clumps. Popular implementations are clumpfind (Williams et al. 1995, 2011), which assigns all emission down to $I_{\min }$, and cprops (Rosolowsky \& Leroy 2006, 2011), which only assigns emission down to the lowest isointensity surface that contains a single local maximum.

An alternate analysis considers isointensity surfaces as a set of hierarchical entities, instead of assigning emission to disjoint clumps. This is more naturally suited to the hierarchical structure in molecular clouds, but a given emitting pixel gets plotted and analyzed multiple times, as part of multiple isointensity structures. The smallest structures associated with local intensity maxima are called "leaves," and the largest isolated regions of emission are called "trunks" or "islands." The most commonly used implementation is dendrograms (Rosolowsky et al. 2008). For clump segmentation, we use the quickclump python implementation (https://github.com/ vojtech-sidorin/quickclump/), to which we added an $I_{\text {minpk }}$ parameter, the minimum peak intensity required for a valid clump (https://github.com/indebetouw/quickclump). This allows the fainter envelopes of bright clumps to be included without keeping faint noise peaks. Properties (moments, fitted sizes, etc.) of clumps are calculated with a python translation of the moments calculations in cpropstoo (https://github.com/akleroy/cpropstoo). For dendrograms we use the python implementation at https://github.com/ dendrograms/astrodendro.

Somewhat more recently it has become popular to identify elongated regions of emission and refer to them as filaments. We use filfinder (Koch \& Rosolowsky 2015) and its python implementation (https://github.com/indebetouw/FilFinder), which allowed for easy modification, testing, and incorporation into other analysis scripts. Filaments are initially identified in the (2D) peak intensity image-if there is not an overabundance of sight lines with multiple velocity components, this should work well, and indeed for these data, the method produces a visually very satisfactory result (Figure 4). Manual examination and searching of this data cube only find one position at which there are two bright structures at different velocities, and fewer than 10 positions with a bright structure at one velocity and structure wings or faint emission at another velocity. filfinder performs pruning of small and multiply connected filament branches. We modified filfinder to do that pruning in $3 \mathrm{D}$, as well as the default $2 \mathrm{D}$. That requires choosing a metric to calculate length in position-positionvelocity space, so we used $1 \mathrm{~km} \mathrm{~s}^{-1} \sim 0.036 \mathrm{pc}$. Our trials found that the final filament skeletons differ only in minor ways between the default $3 \mathrm{D}$ pruning and pruning based on $3 \mathrm{D}$ lengths, so here we present only the default $2 \mathrm{D}$ results using the publicly available code.

It is important to note the bias of the various algorithms: clump segmentation will divide emission into approximately round entities a few times the beam size. Filament finders will identify filament skeletons for any distribution of emission, whether visually filamentary or not. Dendrograms do not impose such geometric constraints but multiply count most emission. To leverage the objective nature of dendrograms in filament analysis, we match filament branches to elongated dendrogram entities. We identify and place greater confidence in those filaments for which there is an isointensity surface that surrounds most of the filament, which contains most of only 

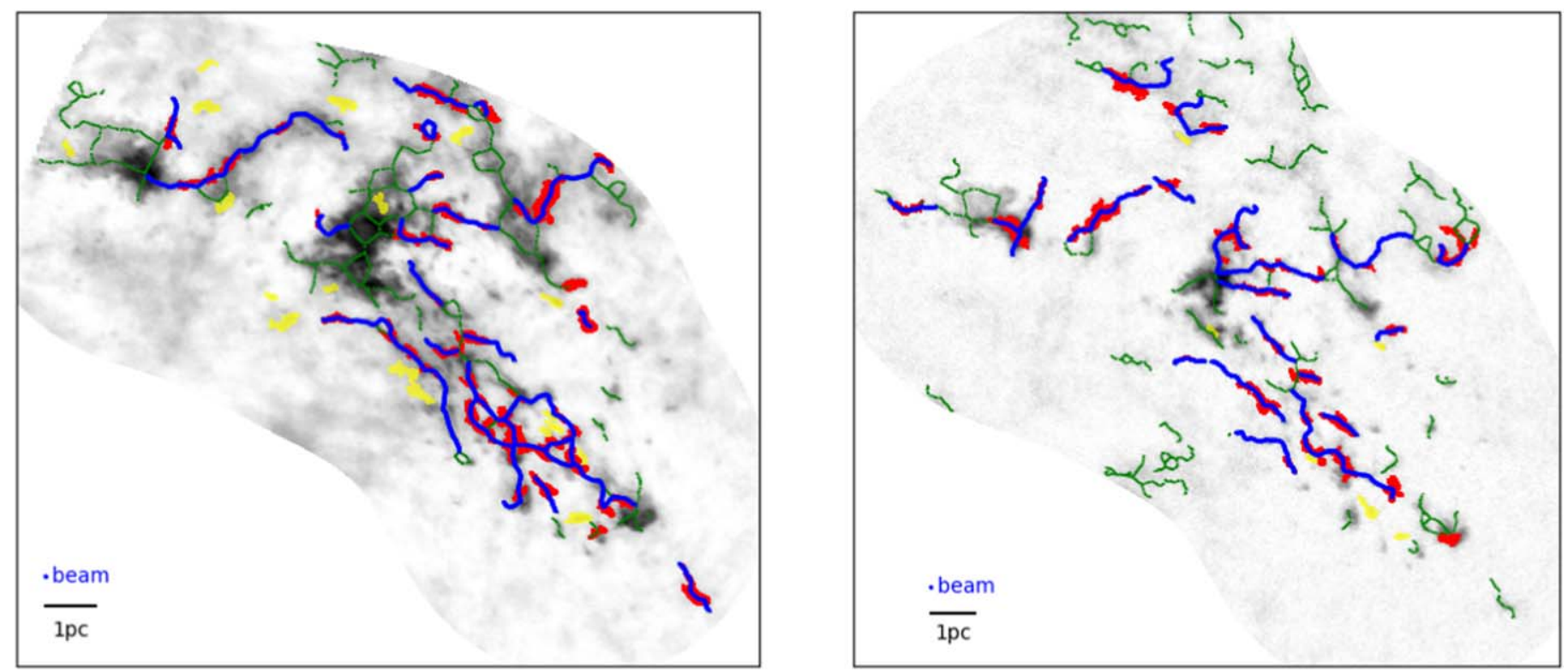

Figure 4. Filaments identified in peak intensity maps of ${ }^{12} \mathrm{CO} 2-1$ (left) and ${ }^{13} \mathrm{CO} 2-1$ (right). Filaments that match with elongated dendrogram structures are shown in blue, with their corresponding dendrogram structures in red. Filaments that do not match elongated dendrogram structures are marked in green. Elongated dendrogram structures that do not match filaments are shown in yellow.

one filament and is elongated (in the end a cutoff aspect ratio $>2.5: 1$ was used, but higher cutoffs up to 5:1 yielded similar results). Figure 4 shows the filfinder filaments identified in the peak intensity image and the elongated isointensity surfaces that match some of the filament branches. Filaments are found using a global threshold of 0.17 and 0.07 times the peak intensity for ${ }^{12} \mathrm{CO}$ and ${ }^{13} \mathrm{CO}$, respectively. The image is flattened at $90 \%$ of peak, smoothed to 3 times the beamwidth, and with size and adaptive thresholds of 24 times the beam area and 14 times the beamwidth used (see filfinder documentation for parameter descriptions). Branches were pruned with thresholds of 5 beams and 10 pixels. Only isointensity structures more elongated than 2.5:1 with areas between 0.2 and $0.5 \mathrm{pc}^{2}$ were matched to filament branches. Overall, there is good but not perfect agreement between identified filaments and elongated isointensity contours, so a filament analysis will reveal different information relative to analyzing all dendrogram structures (defined by isointensity surfaces). In regions where filaments are relatively isolated, the same filaments are identified using ${ }^{12} \mathrm{CO}$ and ${ }^{13} \mathrm{CO}$, although sometimes the ${ }^{13} \mathrm{CO}$ intensity is low enough to cause gaps or breaks in the ${ }^{12} \mathrm{CO}$-identified filament. In dense clumps such as those in the center and east of the region, containing the most massive Spitzer-identified protostars (see Section 3), ${ }^{12} \mathrm{CO}$ becomes very optically thick, and the relatively flat spatial intensity profile over the entire clump causes the filament finder to break the clump up into loops or cells (see especially the central clump with cyan loops in the ${ }^{12} \mathrm{CO}$ image; left panel of Figure 4).

\subsection{Mass Calculation}

Physical analysis of structures identified in clouds requires calculating the mass of each structure. We calculate the mass per PPV pixel from ${ }^{12} \mathrm{CO}$ and ${ }^{13} \mathrm{CO}$ brightness using what is sometimes referred to as the LTE or "standard" method (Bourke et al. 1997; Indebetouw et al. 2013). The ${ }^{12} \mathrm{CO}$ excitation temperature is derived from the ${ }^{12} \mathrm{CO} 2-1$ brightness temperature. The ${ }^{13} \mathrm{CO}$ excitation temperature is assumed to be the same as ${ }^{12} \mathrm{CO} 2-1$, to calculate the ${ }^{13} \mathrm{CO}$ optical depth and column density.

To assess the accuracy of this method, we ran a grid of nonLTE excitation models with Radex (van der Tak et al. 2007), spanning $N\left({ }^{12} \mathrm{CO}\right) \in\left[10^{16}, 10^{21}\right] \mathrm{cm}^{-2}, T_{K} \in[2100] \mathrm{K}, n\left(\mathrm{H}_{2}\right) \in$ $\left[10^{2}, 10^{7}\right] \mathrm{cm}^{-3}$, and $N\left({ }^{12} \mathrm{CO}\right)=75 N\left({ }^{13} \mathrm{CO}\right.$ ) (Heikkilä et al. 1999; Nikolić et al. 2007). From the Radex-computed brightness temperatures for ${ }^{12} \mathrm{CO} 2-1$ and ${ }^{13} \mathrm{CO} 2-1$, we apply the standard calculations:

$$
T_{\mathrm{ex}}=\frac{11.1 K}{\ln \left(\frac{11.1}{I_{12}+0.19}+1\right)},
$$

where $I_{12}$ is the ${ }^{12} \mathrm{CO} 2-1$ intensity in $\mathrm{K}$,

$$
\begin{aligned}
\tau_{0}^{13} & =-\ln \left[1-\frac{T_{B}^{13}}{10.6}\left\{\frac{1}{e^{10.6 / T_{\mathrm{ex}}}-1}-\frac{1}{e^{10.6 / 2.7}-1}\right\}^{-1}\right] \\
N\left({ }^{13} \mathrm{CO}\right) & =1.5 \times 10^{14} \frac{T_{\mathrm{ex}} e^{5.3 / T_{\mathrm{ex}}} \int \tau_{v}^{13} d v}{1-e^{-10.6 / T_{\mathrm{ex}}}} .
\end{aligned}
$$

We considered only models with $N\left({ }^{13} \mathrm{CO}\right)<10^{14} n\left(\mathrm{H}_{2}\right)$, corresponding to line-of-sight path length $<1 \mathrm{pc}$ for an abundance $\mathrm{H}_{2} /{ }^{13} \mathrm{CO}=5 \times 10^{5}$. The results of this section are insensitive to path lengths, and $\mathrm{H}_{2} /{ }^{12} \mathrm{CO} /{ }^{13} \mathrm{CO}$ abundances differ by up to a factor of 3 in either direction. Figure 5 compares $N\left({ }^{13} \mathrm{CO}\right)$ calculated with the LTE method to the actual value input to each model. The method works fairly well, with a tendency to overestimate the true column density in brighter regions. There is a modest effect that the calculated column density (and mass) of bright structures may be overestimated by up to a factor of 2 relative to faint structures.

\subsection{Structure Analysis Result: Filament Velocity Structure}

Part of understanding how stars accrete mass, and how that relates to molecular cloud structure, is determining the extent to which mass is accreted along filaments. To begin to quantify 

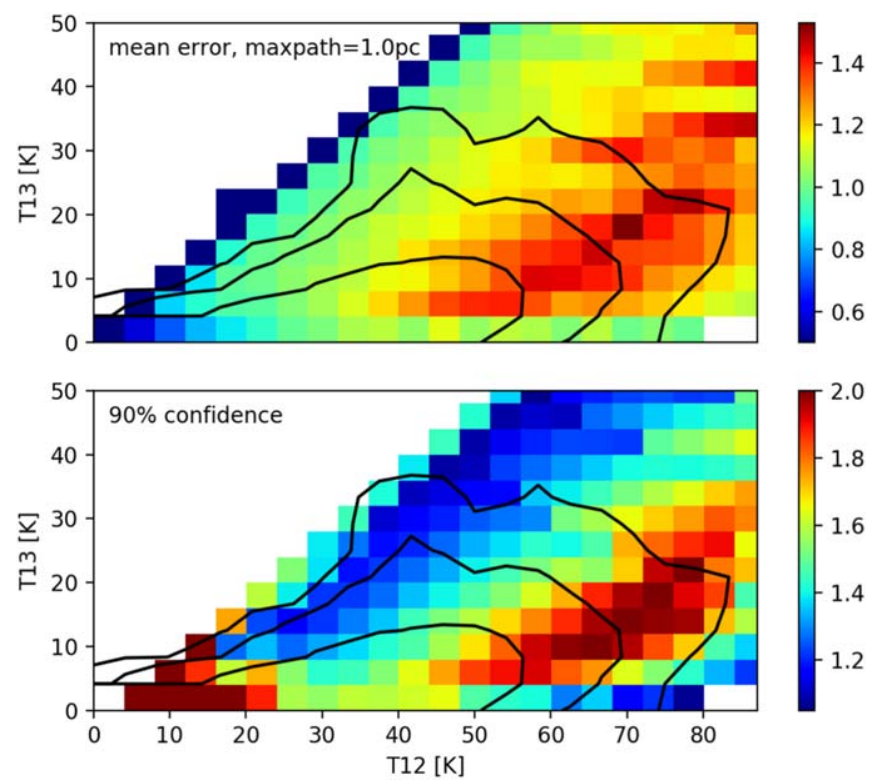

Figure 5. Assessment of validity of the commonly used "LTE method": $N$ $\left({ }^{13} \mathrm{CO}\right)$ calculated from modeled ${ }^{12} \mathrm{CO}$ and ${ }^{13} \mathrm{CO}$ brightness temperatures, compared to the true values input to each model. The top panel is the average $N_{\text {LTE }} / N_{\text {true }}$ for all models with given ${ }^{12} \mathrm{CO} 2-1$ and ${ }^{13} \mathrm{CO} 2-1$ brightness temperatures. The contours are a histogram of the observed values in our cubes. The bottom panel shows the value of the error for which $90 \%$ of the models are closer to the true value. The method works fairly well, overestimating the true column density by up to a factor of 2 at $90 \%$ confidence.

filamentary accretion in this region, we calculated the intensityweighted mean velocity at each point in the map (the first moment of the intensity cube) and then calculated the 2D gradient of that velocity field. The left panel of Figure 6 shows the ${ }^{12} \mathrm{CO} 2-1$ moment 1 velocity map, with filaments overlaid. One diagnostic is whether the velocity gradients are predominantly aligned with filaments, as would be the case if accretion along relatively long-lived filaments were dominant, or across the filaments, as would be the case if the filaments were the result of a turbulent velocity field and not very dynamically important in the cloud. The right panel of Figure 6 shows the distribution of angles between the local gradient in the moment 1 velocity and the filament skeleton direction. No strong trend is evident in the alignment, although there is a weak trend for filaments with a velocity rms larger than $1 \mathrm{~km} \mathrm{~s}^{-1}$ to have a velocity gradient more across than along the filament. This result suggests that accretion along filaments, although it may be present, does not dominate over the stochastic turbulent velocity motions in the cloud.

\subsection{Structure Analysis Result: Filament Stability}

The stability of cylindrical shapes has been calculated for numerous cases, including infinite homogeneous (Chandrasekhar \& Fermi 1953; Ostriker 1964b), polytropic (Ostriker 1964a), and magnetized (Stodólkiewicz 1963; Tilley \& Pudritz 2003). Unmagnetized isothermal filaments with line mass, i.e., mass per unit length $M_{l}>2 \sigma^{2} / G$, are unstable to gravitational collapse, where $\sigma$ is the velocity dispersion and $G$ is the gravitational constant. Other geometries and equations of state change the critical value by a factor of order unity. We calculate the critical line mass at each point of each filament, for only thermal support using the $\mathrm{CO}$ excitation temperature, i.e., $\sigma^{2}=k T_{\mathrm{ex}} / \mu$, where $\mu$ is the mean molecular mass $2.36 m_{H}$, and for thermal and turbulent support $\sigma^{2}=k T_{\mathrm{ex}} / \mu+\sigma_{v}^{2}$, where $\sigma_{v}$ is the second velocity moment of the filament, calculated over the part of the cube within $5 \mathrm{~km} \mathrm{~s}^{-1}$ of the filament peak velocity.

We calculate the actual line mass three ways: for each point on the filament, filfinder extracts the profile perpendicular to that point. We fit a Gaussian of width $\sigma_{r}$ to the profile of ${ }^{12} \mathrm{CO}$ 2-1 intensity and calculate $M_{l}=\sigma_{r} \sqrt{2 \pi} N_{\mathrm{pk}}$ using the LTE column density in the center of the filament at that point, $N_{\mathrm{pk}}$. The fitted widths and peak column densities are calculated from the average of a 3-pixel neighborhood (smaller than the angular resolution) to increase signal-to-noise ratio. In the second method, we fit the width and amplitude of the perpendicular profile in $N$ and use $M_{l}=\sigma_{r} \sqrt{2 \pi} N_{\text {amp. }}$. In the third method, we simply integrate $N$ across each point of the filament. When structures are close together in the direction perpendicular to the filament axis, the width-fitting methods do not have as many points to fit that are unambiguously associated with the given filament and thus can underestimate $\sigma_{r}$ and $M_{l}$, but on the other hand, the integrated $N$ will be an overestimate because it includes unassociated emission. Figure 7 shows the three line masses and two critical masses along one example filament. The more visually fragmented portion of the filament $\sim 1 \mathrm{pc}$ from the bottom (the "gap") has $M_{l} \ll M_{l \text {, crit }}$, and that material is likely not gravitationally bound. By contrast, the bright knot $\sim 1.7 \mathrm{pc}$ from the bottom of the inset (from the left in the profile) appears to be unsupported against collapse, with $M_{l}>M_{\text {crit,nonthermal }}$. Support for other portions of the filament is more ambiguous, with $M_{\text {crit,thermal }}<M_{l}<M_{\text {crit,nonthermal }}$.

The gravitational stability of $0.1 \mathrm{pc}$ scale structures in 30Dor-10 analyzed as filaments is shown in Figure 8. All points for all filaments are now plotted together, after analyzing all filaments as shown in Figure 7 . Points with $M_{l}>M_{\text {crit }}$ have more mass per unit length than thermal or kinetic support can prevent from collapsing (the nonthermal critical mass is shown). The points cluster at $M_{l}=M_{\text {crit }}$, but there are many with $M_{l}>M_{\text {crit }}$ that might be unstable, or supported by magnetic fields. There is a systematic uncertainty in $M_{l}$ depending on the choice of abundance ratio $n\left(H_{2}\right) / n\left({ }^{13} \mathrm{CO}\right)$. A value of $5 \times 10^{6}$ was used here; a lower ratio, or higher ${ }^{13} \mathrm{CO}$ abundance, by a factor of 5 would move most points into the stable part of the plot. Alternately, a factor of 3 lower ratio, combined with a systematic overestimate of $N_{\text {LTE }}$ by a factor of two (Figure 5, Section 4.2), would also bring most points into the stable regime. Given these systematic uncertainties, it may be more illuminating to examine the trends-clearly, locations with brighter ${ }^{12} \mathrm{CO} 2-1$, or those with brighter $8 \mu \mathrm{m}$ emission (from Spitzer/SAGE; Meixner et al. 2006), are statistically less gravitationally stable. Both trends are present when all filament branches are considered (circles), as well as when only considering filaments that are associated with elongated isointensity contours (crosses; see Figure 4 and Section 4.1). It is not unexpected that less stable parts of the cloud might have more significant associated star formation, and hence brighter $8 \mu \mathrm{m}$ emission, but the Spitzer resolution of $>0.5 \mathrm{pc}$ makes it difficult to unambiguously associate emission with $0.1 \mathrm{pc}$ scale molecular structures or to draw strong conclusions.

In Figure 9 we present the width distribution of filaments. The spatial moment of ${ }^{12} \mathrm{CO}$ intensity perpendicular to the filament was calculated at each position, as well as the spatial moment of calculated column density. The weighted mean of those moments is used as the best estimator of the spatial sigma 

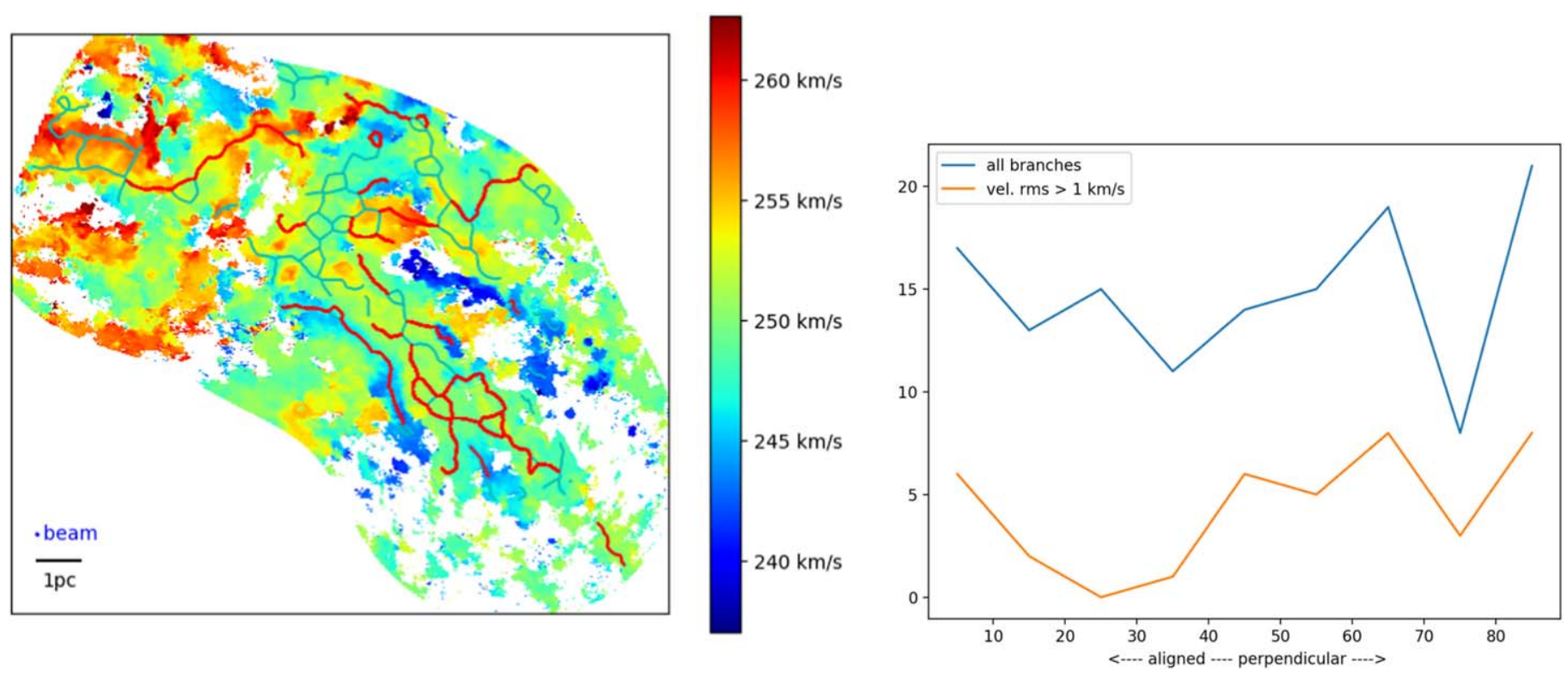

Figure 6. Left: intensity-weighted first moment map of ${ }^{12} \mathrm{CO} 2-1$, with filaments overlaid. At each point, the local gradient in the $2 \mathrm{D}$ velocity field was measured. Right: histogram of the angles between each filament branch's skeleton and the local velocity gradient—an angle of zero would result if the velocity gradient were completely aligned with the filament branch. Histograms are shown for all filament branches and for those that are matched to elongated dendrogram structures. No strong trend is evident in the alignment.

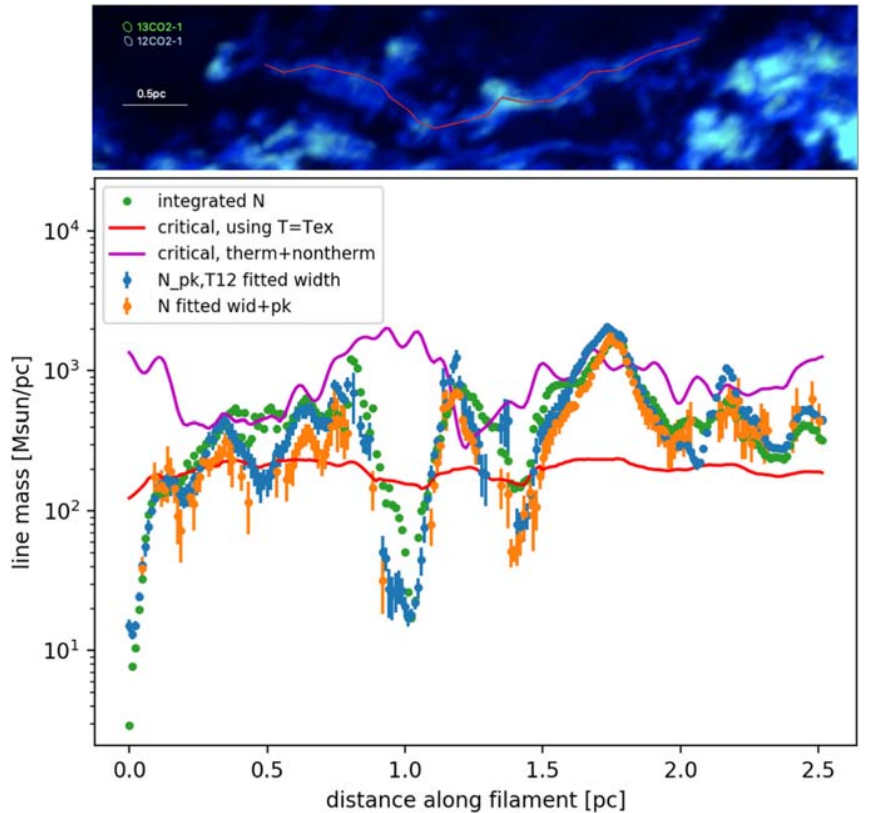

Figure 7. Top: representative filament, showing ${ }^{12} \mathrm{CO}$ and ${ }^{13} \mathrm{CO}$ peak intensity as blue and green. Bottom: line mass calculated three different ways along the filament (green, blue, and orange circles). Critical masses for only thermal support (red) and thermal+nonthermal support (magenta) are also plotted. The more visually fragmented portion of the filament $\sim 1 \mathrm{pc}$ along the filament (the "gap") has $M_{l} \ll M_{\text {crit. }}$. The bright knot $\sim 1.7 \mathrm{pc}$ along appears to be unsupported against collapse, with $M_{l}>M_{\text {crit,nonthermal }}$.

$\sigma_{r}$, and the deconvolved width is $\sqrt{\left(2.354 \sigma_{r}\right)^{2}-0.075^{2}}$. There is no significant difference in the width distribution between points on filaments that have line mass greater than critical line mass including nonthermal (turbulent) support. The median width is $0.09 \mathrm{pc}$, with a standard deviation of $0.06 \mathrm{pc}$, so the filaments in 30Dor-10 have consistent widths to the $0.1 \mathrm{pc}$ width found in solar neighborhood clouds (Arzoumanian et al. 2019).

\subsection{Structure Analysis Result: Size-Line Width-Flux Relations}

The relations between size, velocity dispersion, and surface density (or luminosity as a proxy for mass) have long been studied to yield insight into the structure and gravitational stability of molecular clouds (e.g., Goodman et al. 2009; Heyer et al. 2009; Ballesteros-Paredes et al. 2011; Bolatto et al. 2013). Figure 10 shows the relation for this cloud, for structures segmented with dendrograms and clumpfind, and compared to other molecular clouds. The "radius" plotted is the historical value of 1.91 times the second moment in the plane of the sky, and both that size and the velocity dispersion have had the instrumental resolution deconvolved. The differences between algorithms are clear-the dendrogram analysis classifies all emission, down to very small structures, and multiply counts that emission, as part of very large structures. By contrast, clumpfind is highly biased toward selecting clumps in a narrow range of sizes. The relation is fit to a power law with the Levenberg-Marquardt least-squares fitter in the Kapteyn package (Terlouw \& Vogelaar 2015). The slope of $0.75 \pm 0.05$ is somewhat steeper than the theoretical slope of 0.5 for a medium dominated by turbulent shocks (BallesterosParedes et al. 2011, and references therein), and on the higher side but within $3 \sigma$ of values measured in other clouds (see references and discussion of 30Dor-10 at lower angular resolution in Nayak et al. 2016).

N159, a pair of massive star-forming clouds just to the south of 30 Doradus, has been observed with ALMA at similar angular resolution to the data presented here (Saigo et al. 2017: resolution of 1". $21 \times 0$ ". 84; Tokuda et al. 2019: resolution of 0 ". $29 \times 0$ ". 25 ; Fukui et al. 2015: resolution of 1 !" $3 \times 0$ !" 8 ; Fukui et al. 2019: resolution of 0 !" $28 \times 0$ !" 25 ); dendrogram structures in N159 have a size-line width slope of $0.7 \pm 0.1$, consistent with 30Dor-10, but the line width at a given scale is $0.33 \pm 0.05$ dex lower. The molecular mass of N159 is significantly higher than 30Dor-10, so the enhanced line widths in 30 Doradus cannot be explained by global equilibrium between gravitational and kinetic energy. The 

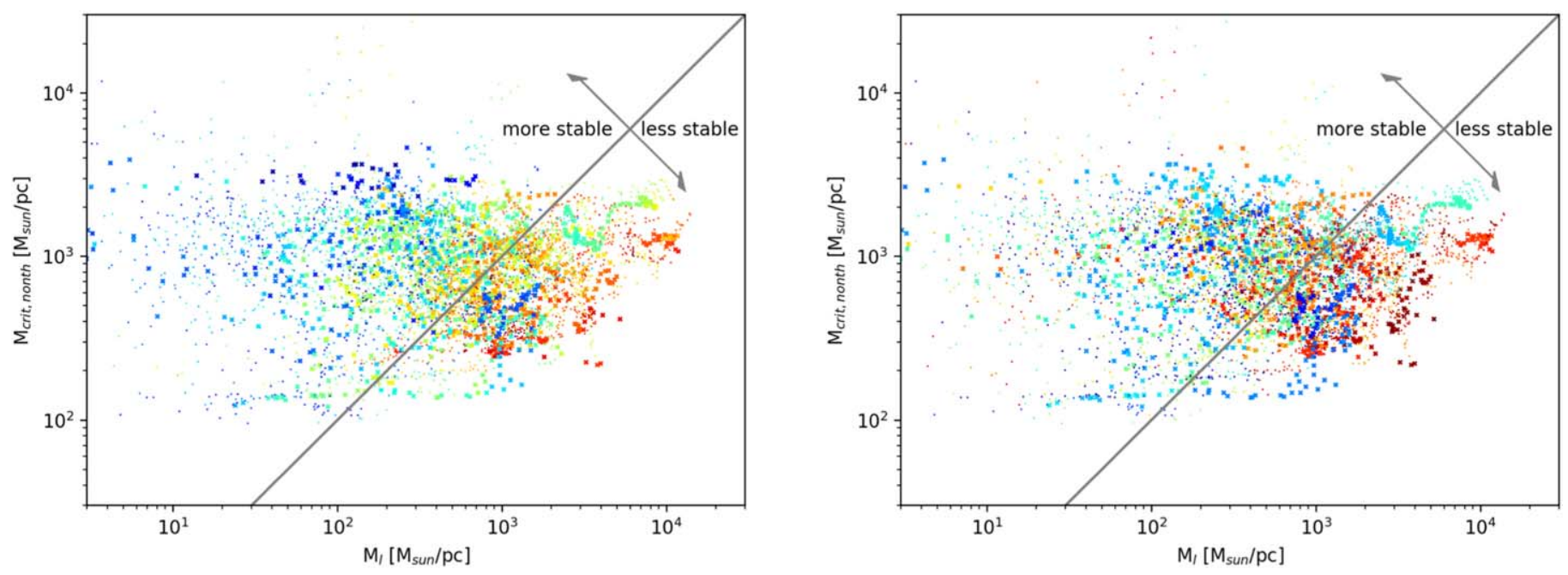

Figure 8. Filament stability: points with $M_{l}>M_{\text {crit }}$ have more mass per unit length than thermal or kinetic support can prevent from collapsing. Left: points colored by ${ }^{12} \mathrm{CO} 2-1$ brightness temperature from lower (blue) to higher (red). Crosses are those filaments that correspond well to elongated isointensity contours (Figure 4). Right: points colored by $8 \mu \mathrm{m}$ brightness from lower (blue) to higher (red).

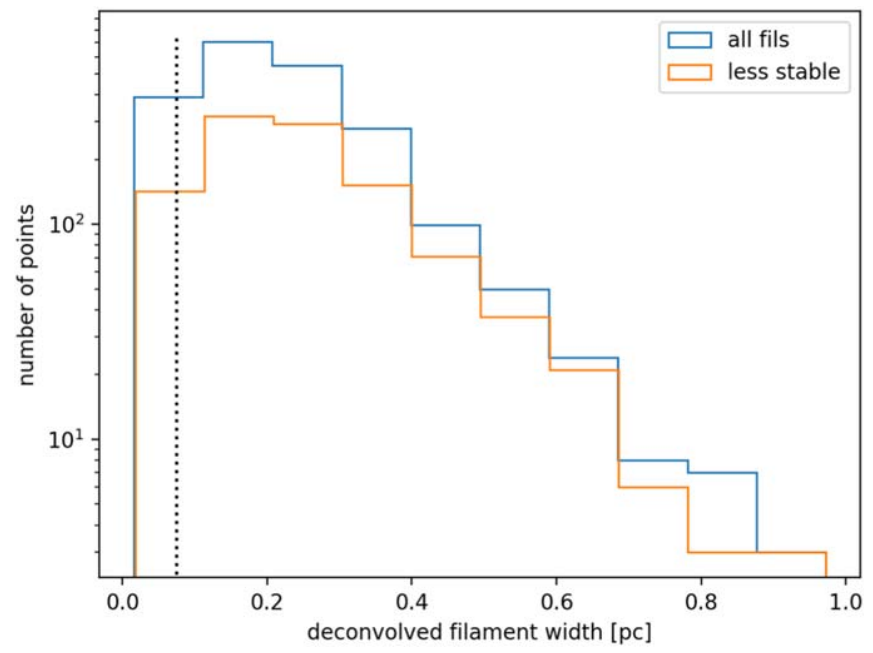

Figure 9. Distribution of widths of positions along filaments, where "width" = 2.534 times the fitted spatial moment of intensity, with the beam size of $0.075 \mathrm{pc}$ subtracted in quadrature. All points with fitted width $>2$ times the width uncertainty are plotted, as well as the subset with $M_{l}>M_{\text {crit,nonth. The }}$ dotted line indicates the beam size.

enhanced kinetic energy is either a result of feedback or an equilibrium including external pressure on the molecular cloud. There is embedded star formation within 30Dor-10, but the total infrared luminosity and inferred star formation rate are again significantly less than in N159. Thus, internal sources of mechanical energy are unlikely the source of elevated turbulence, and instead this is caused by either thermal pressure from the ionized gas or large-scale dynamics of the region. Interestingly, Lee et al. (2019) analyze high-J CO and far-IR line emission at low spatial resolution in 30 Doradus and find that they require significant energy input from low-velocity shocks to explain the CO line spectral energy distribution. They also conclude, based on the distribution of main-sequence and protostellar wind sources, that local kinetic energy injection is unlikely to dominate, but instead they favor kiloparsec-scale energy injection related to the overall dynamics of the region. This kiloparsec-scale energy input is very likely related to kiloparsec-scale colliding filaments that intersect at exactly the center of 30 Doradus, and it may well be the reason that the super star cluster could form there in the first place (Fukui et al. 2017).

Also shown in Figure 10 are the relation fit to Galactic molecular clouds $\delta v=0.72 R^{0.5}$ (Solomon et al. 1987; Heyer et al. 2009; SRBY) and structures in the Perseus A cloud in the solar neighborhood (Ridge et al. 2006). The fitted slope of $0.5 \pm 0.05$ and intercept are consistent with the SRBY relation, with dispersion at a given scale $0.65 \pm 0.05$ lower than in 30Dor-10.

Figure 11 shows the relation between LTE and virial surface density for structures in 30Dor-10. $\Sigma=M / \pi R^{2}$, where $R$ is the cloud "radius" $1.91 \sqrt{\sigma_{x} \sigma_{y}}$ calculated from the weighted spatial moments $\sigma_{x}$ and $\sigma_{y}$. One can also use the "exact area" or full spatial extent of the pixels assigned to each structure-this area is 1.2-1.9 times larger, decreasing both surface densities accordingly. $\Sigma_{\text {LTE }}$ is calculated from the LTE mass, and $\Sigma_{\text {vir }}$ from the virial mass $5 \sigma_{v}^{2} R / G$. This plot is sometimes referred to as a "boundedness" plot because it probes the degree to which kinetic energy is gravitationally bounded. Structures in virial equilibrium between gravitational and kinetic energy would lie along $\Sigma_{\mathrm{vir}}=\Sigma_{\mathrm{LTE}}$, and those in freefall (hierarchical) collapse driven by gravity along $\Sigma_{\text {vir }}=2 \Sigma_{\text {LTE }}$ (Ballesteros-Paredes et al. 2011). Alternately, clumps might be in virial equilibrium between kinetic and gravitational energy with external pressure (Field et al. 2011), if they are sufficiently long-lived to achieve that virialization (Bonnell et al. 2006). The resulting curves

$$
\Sigma_{\mathrm{vir}}=\Sigma_{\mathrm{LTE}}+\frac{20}{3 \pi 20.9} \frac{n T}{\Sigma_{\mathrm{LTE}}}
$$

are marked in Figure 11 for external pressures of $n T=$ $\left(10^{5}, 10^{6}\right) \mathrm{cm}^{-3} \mathrm{~K}$.

Structures segmented using clumpfind fall in a similar locus to much larger (1-10 pc) molecular clouds in the Milky Way disk and Galactic central molecular zone (Oka et al. 2001; Heyer et al. 2009). These all fall somewhat above the unity relation expected from virial equilibrium and are more consistent with either external pressure confinement or freefall collapse. Structures in 30Dor-10 lie in between the disk and central molecular zone (CMZ) clouds; if the relevant model is 

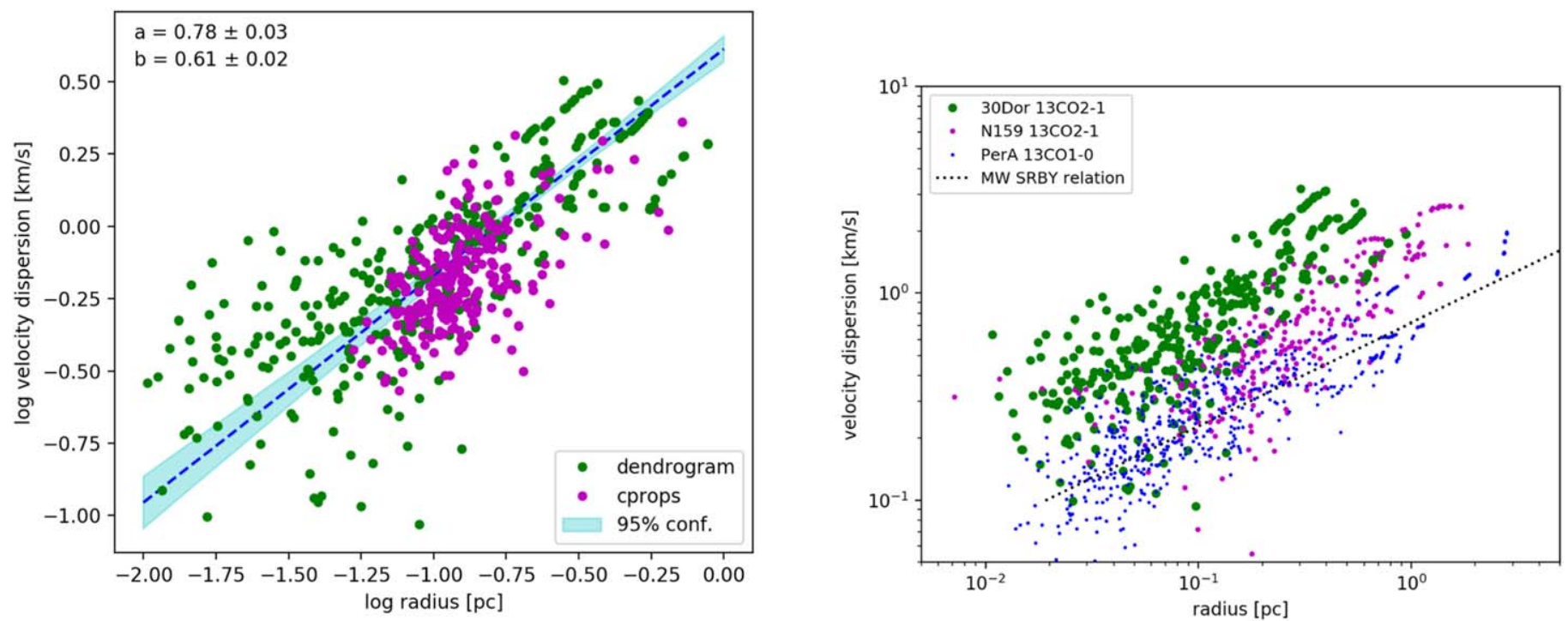

Figure 10. Left: size-line width relation in 30Dor-10 for dendrogram (green) and clumpfind (magenta) structures. Overlaid is the slope fitted to dendrogram structures $\log \left(\sigma_{v}\right)=a \log (r)+b$. Right: comparison of size-line width relations between 30Dor-10, the nearby less evolved LMC massive star formation region N159, Galactic molecular cloud Perseus A, and the "historic" Milky Way disk relation (dashed line; the data from which that was derived are all off the right-hand side of the plot; Solomon et al. 1987; Heyer et al. 2009). Data from all regions were reanalyzed the same way, with clumps segmented using dendrograms.

confinement by external pressure $P_{e}$, then that pressure is higher in 30Dor-10 than typical Milky Way but lower than the CMZ. For the large Milky Way clouds, $P_{e}$ should be interpreted as pressure from the neutral interstellar medium surrounding the molecular cloud, but for the subparsec structures plotted in 30Dor-10, $P_{e}$ is the pressure external to a core, which is a combination of any warm interstellar medium pressure acting on the entire cloud and the effective pressure of the diffuse cloud acting on the core. In Paper I we used the formula of Bertoldi \& McKee (1992) to estimate that interclump pressure to be $3 \times 10^{6} \mathrm{~cm}^{-3} \mathrm{~K}$, consistent with the location of core and clumps in Figure 11.

Structures identified by dendrogram have significantly higher $\Sigma_{\text {LTE }}$ than those identified with clumpfind. This conclusion is unchanged if either the "exact" cloud area is used to calculate $\Sigma$ or observed quantities (without the velocity and angular resolution deconvolved) are plotted. Dendrogram structure properties are calculated in Figure 10 by assuming that all emission above each isointensity surface is part of the structure (bijection), but one could also subtract the value of that lower bounding surface (clipping), as discussed in Rosolowsky \& Leroy (2006). Clipping decreases $\Sigma_{\text {vir }}$ by only 0.02 dex, while decreasing $\Sigma_{\text {LTE }}$ by 0.3 dex-the mean difference of $\Sigma_{\text {LTE }}$ between dendrogram and clumpfind structures is $0.8 \mathrm{dex}$ without clipping and decreases to $0.5 \mathrm{dex}$ when clipping is used. Rosolowsky \& Leroy (2006) conclude that clipping underestimates clump masses for their solar neighborhood clouds, but for the data presented here, clipping would result in structures with $\Sigma_{\mathrm{LTE}}$ closer to those identified by clumpfind.

There is some tendency for larger dendrogram structures to be evidently less gravitationally stable: the right panel of Figure 11 shows $\Sigma_{\text {vir }}$ and $\Sigma_{\text {LTE }}$ colored by structure size. The virial mass was calculated for a constant-density sphere, but molecular cloud substructures are clearly not spheres. Generalizing from spheres, the gravitational energy of an ellipsoid can be written in closed form for various radial density profiles
(Neutsch 1979), including constant density,

$$
\begin{gathered}
U=\frac{3}{5} \frac{G M^{2}}{l} w(\eta) \\
w(\eta)=\frac{\eta \sinh ^{-1}\left(\sqrt{\eta^{2}-1}\right)}{\sqrt{\eta^{2}-1}},
\end{gathered}
$$

where $M$ is the mass, $l$ the semimajor axis, and $\eta=l / R$ the aspect ratio (Lee et al. 2017). For the same mass, a more elongated cloud has greater gravitational energy by a factor of $(2,3)$ for a cloud aspect ratio of $\sim(3.5,10)$. Thus, the virial mass required for gravitational energy to balance a given kinetic energy is lower by a factor of a few for an elongated cloud compared to the assumed spherical shape. If we applied this correction, we would conclude that the clouds are even less gravitationally stable and located even lower down in Figure 11. We measured the elongation or aspect ratio of each structure in the figure, but there is no clear trend with boundedness, and applying an elongation correction does not decrease the scatter of points in the plot.

Another source of systematic uncertainty was mentioned in Section 4.4: a factor of 5 higher assumed ${ }^{13} \mathrm{CO}$ abundance would move most dendrogram points into the stable part of the plot. Alternately, a factor of 3 lower ratio, combined with a systematic overestimate of $N_{\text {LTE }}$ by a factor of two (Figure 5), would also bring most points into the stable regime. However, either of those corrections would cause the clumpfind clumps to disagree significantly with previous measurements of molecular clouds in this and many other regions.

The most likely cause for the difference between the boundedness of clumpfind and dendrogram segmented structures is that the dendrogram structures by design do not include entire clumps. Consider a model core with powerlaw density distribution outside of a core radius $r_{c}$. The density distribution $\rho \propto r^{-1}$, for example, was used in Solomon et al. (1987) to derive the commonly used relation between the second spatial moment $\sigma_{x}$ and the "edge" or "effective radius" 

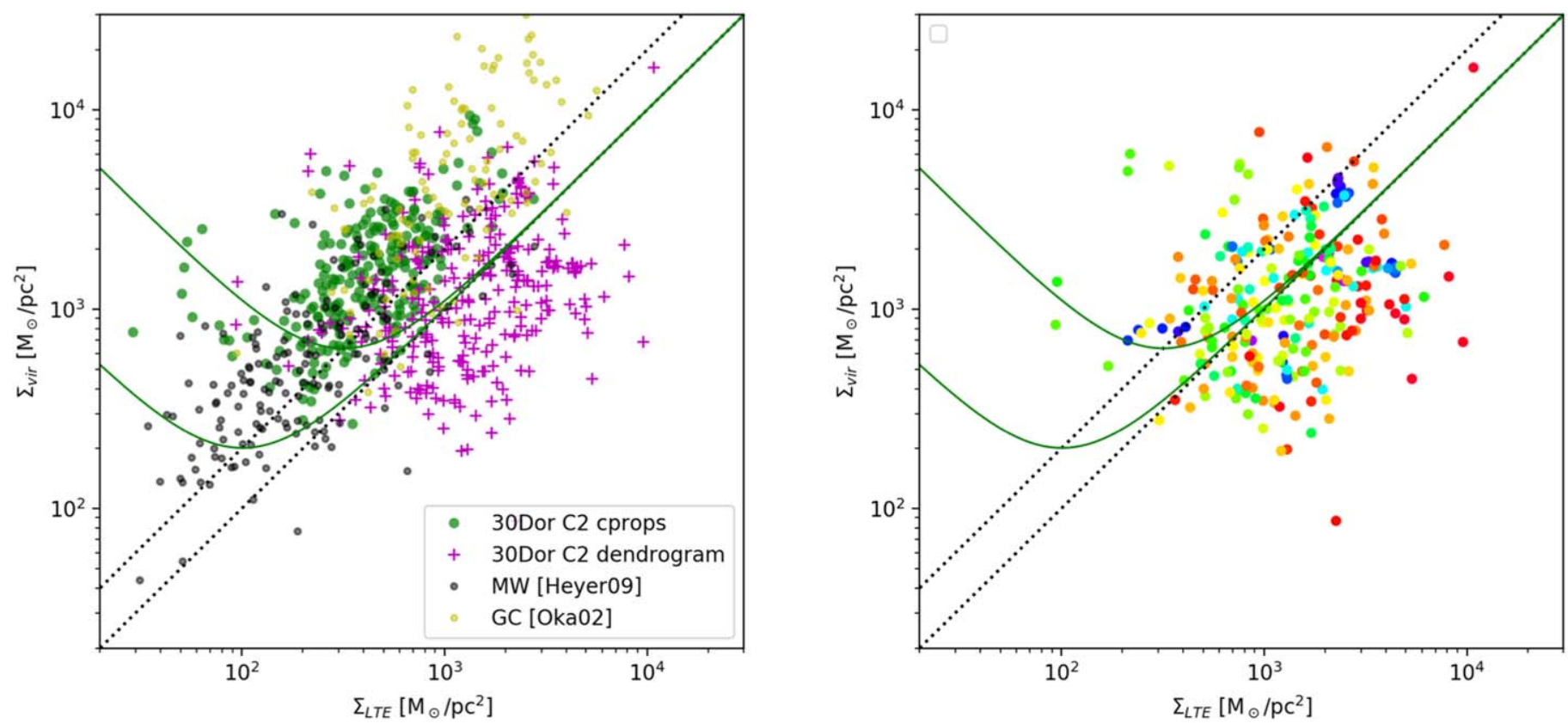

Figure 11. Relation between virial surface density $5 \sigma_{v}^{2} /(G \pi R)$ and LTE surface density $M_{\mathrm{LTE}} /\left(\pi R^{2}\right)$. Virial equilibrium between gravitational and kinetic energy $\Sigma_{\mathrm{vir}}=\Sigma_{\mathrm{LTE}}$ and gravity-driven collapse $\Sigma_{\mathrm{vir}}=2 \Sigma_{\mathrm{LTE}}$ are marked as dashed lines. Solid green curves show the virial equilibrium with external pressures $n T=\left(10^{5}, 10^{6}\right) \mathrm{cm}^{-3} \mathrm{~K}$. Left: different segmentation methods in this region (green = cprops; magenta = dendrogram) are compared to previous measurements of the galactic center (GC; Oka et al. 2001) and Milky Way disk (MW; Heyer et al. 2009). Right: only dendrogram structures in this region are shown, now colored by size from smaller $=$ blue to larger $=$ red.

of the core $R_{e}=1.91 \sigma_{x}$. If one assumes that the velocity dispersion $\sigma_{v}$ follows a power-law size-line width relation $\sigma_{v} \propto R^{\alpha}$, where $R$ is the projected radius in the plane of the sky, then one can calculate the virial surface density

$$
\Sigma_{\mathrm{vir}}(R)=\frac{5 \sigma_{v}^{2}}{\pi G M(R) R_{\text {measured }}}
$$

as a function of projected radius $R$, where $M(R)=$ $\int_{0}^{R} 4 r \rho(r) d r$. The measured radius $R_{\text {measured }}$ is what one could measure from the data within a radius $R$, and it could be simply $R$ itself (i.e., the radius of the assignment area $\sqrt{\operatorname{area} / \pi}$ ), or more commonly the "Solomon effective radius" calculated from the two spatial second moments of the emission, 1.91 $\sqrt{\sigma_{x} \sigma_{y}}$. Figure 12 shows the virial surface density $\Sigma_{\mathrm{vir}}$ and the actual measured surface density $\Sigma=M / \pi / R_{\text {measured }}^{2}$, as a function of $R$ for a model core with two different size-line width relations $\alpha$ and two different power-law density profiles. Clearly, if one calculates a surface density for only the brightest part of a core, one will measure a significantly higher surface density $\Sigma$ than for the entire core. At the same time, the calculated virial surface density $\Sigma_{\text {vir }}$ will be somewhat lower than the whole-core value. This underscores the need to use the same segmentation method when comparing different data sets. It also suggests that if parts of clouds segmented with dendrograms are analyzed, the relative boundedness between two different clouds is a robust comparison, but the absolute value of that boundedness relative to the theoretical lines of stability should be interpreted cautiously. As a final test, we calculated the size-line width-mass relations for structures identified with clumpfind but using a higher and higher noise floor or cutoff, raising it gradually up to $20 \times$ the noise level in

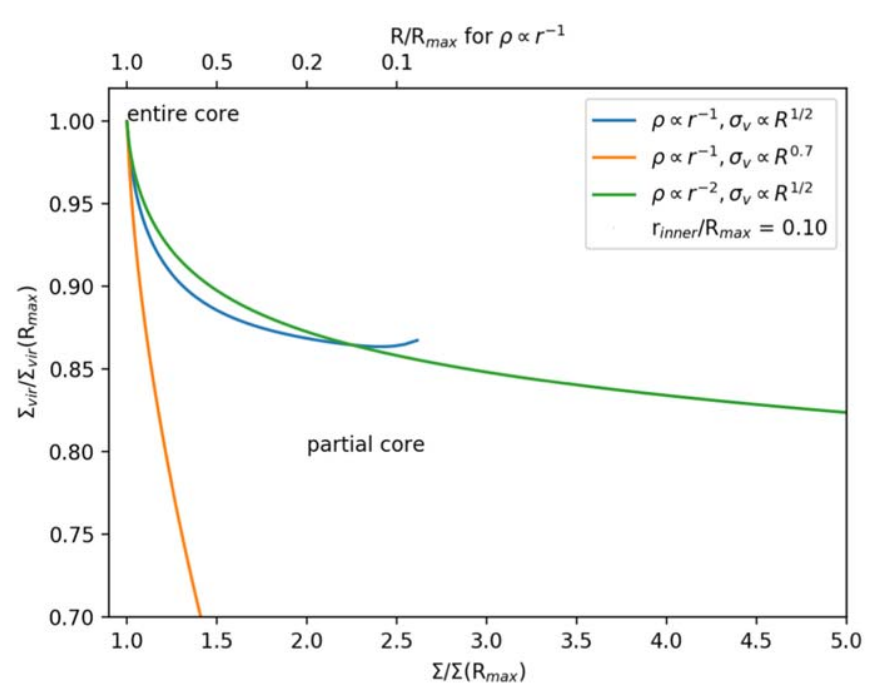

Figure 12. Relation between virial surface density $5 \sigma_{v}^{2} /(G \pi R)$ and LTE surface density $M_{\mathrm{LTE}} /\left(\pi R^{2}\right)$ for a model spherical core. The core has constant density within $r_{\text {inner }}$ and a power-law radial density distribution outside of that. Each part of the core within a projected radius $R$ is assumed to follow the sizeline width relation $\sigma_{v} \propto R^{\alpha}$. Calculation of the two surface densities for a partial core will yield significantly higher $\Sigma$ and moderately lower $\Sigma_{\text {vir }}$ compared to the values calculated for the entire core.

the cube. Raising the cutoff floor causes the assigned structures' properties to smoothly move over to the location of the dendrogram-assigned structures, as expected.

\subsection{Structure Analysis Result: Core Mass Function}

A fundamental question in star formation is whether the stellar IMF is predetermined by the molecular cloud structure. Similarity between the dense core mass function and the IMF would support that premise. We analyze compact structures 

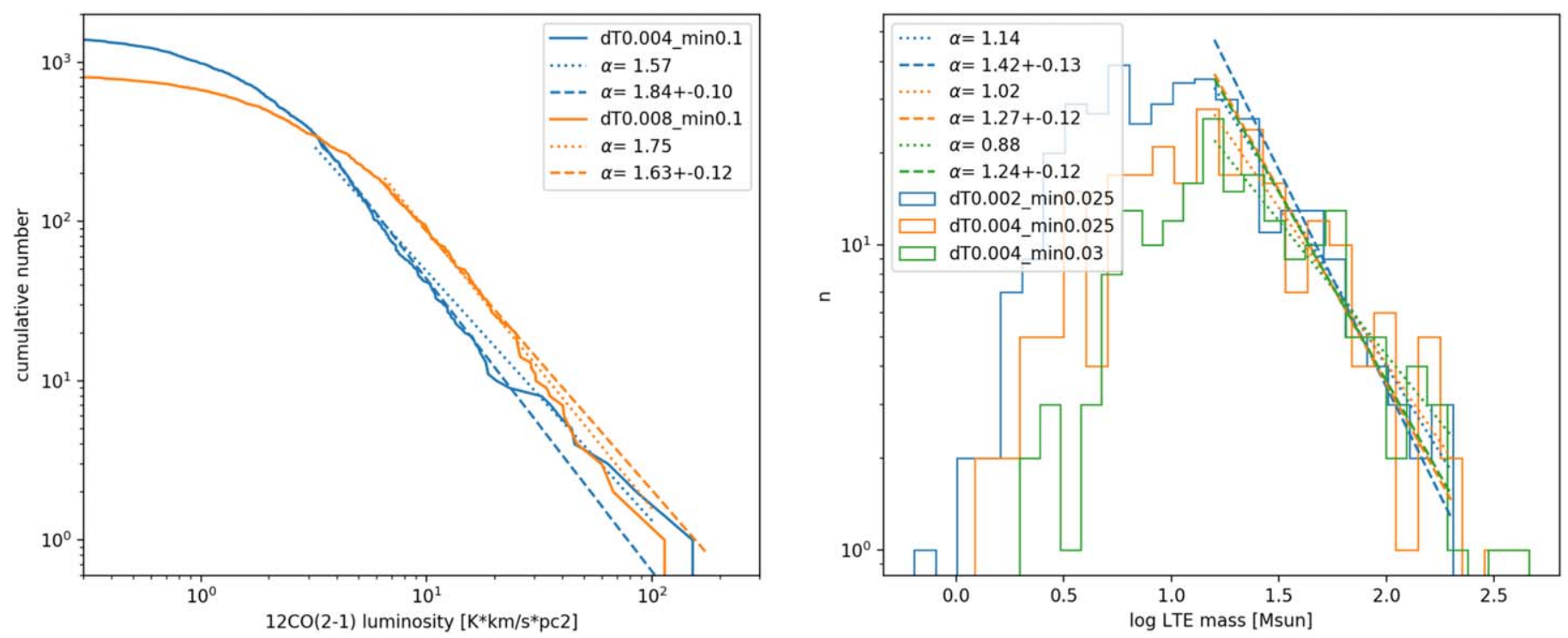

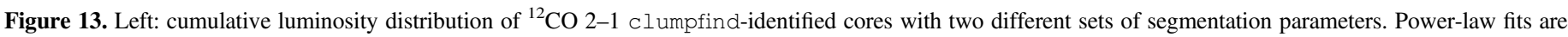

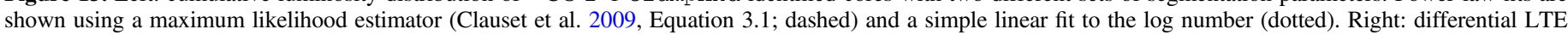
mass function of cores with three different segmentation parameters.

identified using clumpfind (as implemented in cprops). The distinction between calling a molecular cloud structure a clump or core is inhomogeneous in the literature, with $\sim 1 \mathrm{pc}$ structures usually called clumps and $\sim 0.1$ pc structures usually called cores; these structures are $0.1-0.2 \mathrm{pc}$ in diameter, so we call them cores for brevity, without intending any implication about stability or concentration.

Figure 13 shows the core luminosity and mass distributions. Different values of the clumpfind segmentation parameters are shown- $d T$ and $T_{m i n}$, the difference between each local maximum and nearest saddle, and minimum level to analyze. The main effect of varying those parameters is to increase the number of faint cores when $\mathrm{T}_{\mathrm{min}}$ is decreased. Fitting powerlaw distributions has well-studied uncertainties (e.g., Clauset et al. 2009; Maíz Apellániz 2009); we show both the Hill maximum likelihood estimator with its statistical uncertainty (dashed lines) and a simple linear fit to the $\log$ number $n$, weighted by $n^{-0.3}$ (dotted lines; the exponent of the weight makes little difference to the result). It is evident that even with the mathematical uncertainties due to fit method, the uncertainties due to how the emission is segmented into structures are even larger.

${ }^{12} \mathrm{CO}$ luminosity is proportional to cloud mass on large (>parsec) scales (e.g., Bolatto et al. 2013). Although one would expect the luminosity of an optically thick line to be a less reliable mass tracer on small scales when emission fills the beam, it is still interesting to consider the shape of the ${ }^{12} \mathrm{CO}$ luminosity function (Figure 13, left panel). The bright end is fit with a power law of slope $\alpha=1.7 \pm 0.2$. If luminosity were proportional to mass, this would imply a core differential mass function $N(>M) \propto M^{-\alpha}$ slope somewhat steeper than the stellar IMF slope of -1.35 . However, the ${ }^{12} \mathrm{CO}$ optical depth increases systematically with mass $\tau \sim M_{\mathrm{LTE}}^{0.3}$, so the observed ${ }^{12} \mathrm{CO}$ luminosity function is expected to be steeper than the mass function by a slope of about 0.3 , and the inferred mass function from ${ }^{12} \mathrm{CO}$ alone would then be consistent with the IMF, within uncertainties. The right panel of the figure shows the differential LTE mass distribution (from ${ }^{12} \mathrm{CO}$ and ${ }^{13} \mathrm{CO}$ ), for different core segmentation parameters. Slopes are fitted directly to the differential mass distribution, as well as using the aforementioned Hill maximum likelihood estimator. The systematic biases of direct fitting are more evident than when fitting the cumulative distribution. The best estimate slope of $-1.3 \pm 0.15$ is consistent with the stellar IMF slope of -1.35 .

The upper end of the "core" mass distribution is consistent with a power law with the same slope as the stellar IMF. A shallower mass function (more massive stars) has been found for the main-sequence massive stars in 30 Doradus (Schneider et al. 2018). Either the next generation of star formation in 30Dor-10 will not have that massive stellar excess, or the stellar mass distribution is not predetermined by the current core mass function. The discussion of stability above raises the natural question of whether the clumps and cores whose mass distribution is being analyzed should have any correspondence with the stellar mass function, especially if many of those cores are not gravitationally bound or collapsing. To test this, we analyzed the distribution of only cores with $\Sigma_{\text {virial }} / \Sigma$ less than a threshold (we tried thresholds between 2 and 4; see Figure 11 and discussion in Section 4.5 about the normalization of $\Sigma_{\text {virial }} / \Sigma$ ). Interestingly, the shape of the mass distribution and the fitted slope of the upper end are the same for the more gravitationally bound subset(s) of cores. If the mass distribution of cores is independent of core stability and is related to the stellar IMF, then this result supports the notion that the core and cloud structure predetermines the stellar IMF, before gravitational forces become dominant.

\section{Conclusions}

High-resolution $(<0.1 \mathrm{pc})$ observations of the 30Dor-10 molecular cloud $15 \mathrm{pc}$ north of R136 are presented. The CO emission morphology contains clumps near the locations of known mid-IR massive protostars, as well as a series of parseclong filaments oriented almost directly toward R136, most of which do not show signs of embedded star formation. The aligned filaments could possibly be "pillars" left behind by photoionization, or they could be a relic of the initial collapse and formation of 30 Doradus and the R136 star cluster. 
Analysis of the cloud substructures is performed by segmenting emission into disjoint approximately round "cores" using clumpfind, by considering the hierarchical structures defined by isointensity contours using dendrograms, and by segmenting into disjoint long thin "filaments" using Filfinder. The balance between gravitational and kinetic energy of "cores" and dendrogram branches are analyzed with formulae appropriate to spheres and ellipsoids, and the balance is analyzed for filaments using formulae appropriate to infinite cylinders. We find that the filaments have widths of $\sim 0.1 \mathrm{pc}$, similar to those in solar neighborhood clouds.

There is elevated kinetic energy (line widths at a given size scale) in 30Dor-10 compared to other LMC and Galactic star formation regions. The slope of the size-line width relation is also a bit steeper than on those other regions, although not dramatically so. A steeper slope (more energy at larger scales) and a lack of correlation with local sources of kinetic energy (stellar winds and protostellar outflows) suggest that energy is injected on large (hundreds of parsec to kiloparsec) scales. This agrees with the analysis of high- $J$ CO and far-IR line emission at low spatial resolution in 30 Doradus (Lee et al. 2019) and the existence of kiloparsec-scale colliding filaments in the region (Fukui et al. 2017).

Clumps and cores, when analyzed as entire objects with clumpfind, lie in a similar part of size-line width-mass parameter space to the Milky Way and other molecular clouds and are consistent with freefall collapse or virial equilibrium with moderate external pressure. A significant fraction of dendrogram structures and filaments have mass surface densities $\Sigma_{\mathrm{LTE}}$ or line masses $M_{l}$ in excess of the corresponding quantities if gravitational and kinetic energies were in balance. The discrepancy can be resolved if the ${ }^{13} \mathrm{CO} / \mathrm{H}_{2}$ abundance is a factor of 5 or more higher than the value that was assumed here. Alternately, one physical explanation could be that the small-scale structures and filaments in this cloud have significant magnetic support against gravity.

The upper end of the "core" mass distribution is consistent with a power law with the same slope as the stellar IMF. Slopes are fitted directly to the differential mass distribution, as well as using the aforementioned Hill maximum likelihood estimator. The systematic biases of direct fitting are more evident than when fitting the cumulative distribution. The best estimate slope of $-1.3 \pm 0.15$ is consistent with the stellar IMF slope of -1.35 . The shape of the mass distribution including the fitted slope does not change if only a subset of cores that have large $\Sigma / \Sigma_{\text {vir }}$ are considered; the cores that are more likely to collapse and form stars according to our stability measurement do not have a statistically different mass distribution than those that are less likely to collapse. This fact, along with the fact that the most reliably measured part of the mass distribution has the same slope as the stellar IMF, supports the notion that the stellar IMF is predetermined by the characteristics of turbulent fragmentation in the pre-collapse molecular cloud. A shallower mass function (more massive stars) has been found for the main-sequence massive stars in 30 Doradus (Schneider et al. 2018). Either the next generation of star formation in 30Dor-10 will not have that massive stellar excess, or the stellar mass distribution is not predetermined by the current core mass function.

This paper makes use of the following ALMA data: ADS/JAO. ALMA\#2011.0.00471.S, \#2011.0.00471.S, and 2013.1.00346.S.
ALMA is a partnership of ESO (representing its member states), NSF (USA), and NINS (Japan), together with NRC (Canada) and NSC and ASIAA (Taiwan), in cooperation with the Republic of Chile. The Joint ALMA Observatory is operated by ESO, AUI/ NRAO, and NAOJ. The National Radio Astronomy Observatory is a facility of the National Science Foundation operated under cooperative agreement by Associated Universities, Inc. This research has made use of NASA's Astrophysics Data System Bibliographic Services. R.I. was partially supported during this work by NSF AST-1312902.

Software: CASA (v4.3.1; v4.5; v5.1.0 McMullin et al. 2007), Kapteyn (Terlouw \& Vogelaar 2015), filfinder (Koch \& Rosolowsky 2015), quickclump (https://github.com/vojtechsidorin/quickclump/), clumpfind (Williams et al. 1995, 2011), cprops (Rosolowsky \& Leroy 2006, 2011), dendrograms (Rosolowsky et al. 2008)

\section{ORCID iDs}

Rémy Indebetouw (i) https://orcid.org/0000-0002-4663-6827 Tony Wong (i) https://orcid.org/0000-0002-7759-0585 Amanda Kepley (i) https://orcid.org/0000-0002-3227-4917 Vianney Lebouteiller (10) https://orcid.org/0000-00027716-6223

Suzanne Madden (iD https://orcid.org/0000-0003-3229-2899 Joana M. Oliveira (iD https://orcid.org/0000-0002-0861-7094

\section{References}

André, P. 2015, HiA, 16, 31

Arzoumanian, D., André, P., Könyves, V., et al. 2019, A\&A, 621, A42 Ballesteros-Paredes, J., Hartmann, L. W., Vázquez-Semadeni, E., Heitsch, F., \& Zamora-Avilés, M. A. 2011, MNRAS, 411, 65

Bertoldi, F., \& McKee, C. F. 1992, ApJ, 395, 140

Bolatto, A. D., Wolfire, M., \& Leroy, A. K. 2013, ARA\&A, 51, 207

Bonnell, I. A., Dobbs, C. L., Robitaille, T. P., \& Pringle, J. E. 2006, MNRAS, 365,37

Bourke, T. L., Garay, G., Lehtinen, K. K., et al. 1997, ApJ, 476, 781

Brogan, C. L., Goss, W. M., Lazendic, J. S., et al. 2004, AJ, 128, 700 Chandrasekhar, S., \& Fermi, E. 1953, ApJ, 118, 113

Clauset, A., Shalizi, R., \& Newman, M. E. J. 2009, SIAMR, 51, 661 Dale, J. E., Haworth, T. J., \& Bressert, E. 2015, MNRAS, 450, 1199 de Grijs, R., Wicker, J. E., \& Bono, G. 2014, AJ, 147, 122

Elitzur, M., Hollenbach, D. J., \& McKee, C. F. 1989, ApJ, 346, 983

Fernández-López, M., Arce, H. G., Looney, L., et al. 2014, ApJL, 790, L19

Field, G. B., Blackman, E. G., \& Keto, E. R. 2011, MNRAS, 416, 710

Fukuda, N., Hanawa, T., \& Sugitani, K. 2002, ApJL, 568, L127

Fukui, Y., Harada, R., Tokuda, K., et al. 2015, ApJL, 807, L4

Fukui, Y., Tokuda, K., Saigo, K., et al. 2019, ApJ, 886, 14

Fukui, Y., Tsuge, K., Sano, H., et al. 2017, PASJ, 69, L5

Goodman, A. A., Pineda, J. E., \& Schnee, S. L. 2009, ApJ, 692, 91

Gray, M. D., Field, D., \& Doel, R. C. 1992, A\&A, 262, 555

Gritschneder, M., Burkert, A., Naab, T., \& Walch, S. 2010, ApJ, 723, 971

Gruendl, R. A., \& Chu, Y.-H. 2009, ApJS, 184, 172

Heikkilä, A., Johansson, L. E. B., \& Olofsson, H. 1999, A\&A, 344, 817

Heyer, M., Krawczyk, C., Duval, J., \& Jackson, J. M. 2009, ApJ, 699, 1092

Imai, H., Katayama, Y., Ellingsen, S. P., et al. 2013, MNRAS, 432, L16

Indebetouw, R., Brogan, C., Chen, C.-H. R., et al. 2013, ApJ, 774, 73

Johansson, L. E. B., Greve, A., Booth, R. S., et al. 1998, A\&A, 331, 857

Kepley, A., Tsutsumi, T., Brogan, C., et al. 2020, PASP, submitted, arXiv: 1912.04970

Koch, E. W., \& Rosolowsky, E. W. 2015, MNRAS, 452, 3435

Lee, M.-Y., Madden, S. C., Le Petit, F., et al. 2019, A\&A, 628, A113

Lee, Y.-N., Hennebelle, P., \& Chabrier, G. 2017, ApJ, 847, 114 Maíz Apellániz, J. 2009, Ap\&SS, 324, 95

McMullin, J. P., Waters, B., Schiebel, D., et al. 2007, Astronomical Data Analysis Software and Systems XVI, in ASP Conf. Ser. 376, ed.

R. A. Shaw, F. Hill, \& D. J. Bell (San Francisco, CA: ASP), 127

Meixner, M., Gordon, K. D., Indebetouw, R., et al. 2006, AJ, 132, 2268

Motte, F., Bontemps, S., \& Louvet, F. 2018, ARA\&A, 56, 41

Nayak, O., Meixner, M., Indebetouw, R., et al. 2016, ApJ, 831, 32 
Neutsch, W. 1979, A\&A, 72, 339

Nikolić, S., Garay, G., Rubio, M., \& Johansson, L. E. B. 2007, A\&A, 471, 561

Oka, T., Hasegawa, T., Sato, F., et al. 2001, ApJ, 562, 348

Ostriker, J. 1964a, ApJ, 140, 1056

Ostriker, J. 1964b, ApJ, 140, 1529

Peimbert, A. 2003, ApJ, 584, 735

Poglitsch, A., Krabbe, A., Madden, S. C., et al. 1995, ApJ, 454, 293

Rahner, D., Pellegrini, E. W., Glover, S. C. O., et al. 2018, MNRAS, 473, L11

Ridge, N. A., Di Francesco, J., Kirk, H., et al. 2006, AJ, 131, 2921

Rosolowsky, E., \& Leroy, A. 2006, PASP, 118, 590

Rosolowsky, E., \& Leroy, A. 2011, CPROPS: Bias-free Measurement of Giant Molecular Cloud Properties, Astrophysics Source Code Library, ascl:1102.012

Rosolowsky, E. W., Pineda, J. E., Kauffmann, J., \& Goodman, A. A. 2008, ApJ, 679, 1338

Rubio, M., Barbá, R. H., Walborn, N. R., et al. 1998, AJ, 116, 1708

Russell, S. C., \& Dopita, M. A. 1992, ApJ, 384, 508

Sabbi, E., Anderson, J., Lennon, D. J., et al. 2013, AJ, 146, 53

Sabbi, E., Lennon, D. J., Anderson, J., et al. 2016, ApJS, 222, 11
Saigo, K., Onishi, T., Nayak, O., et al. 2017, ApJ, 835, 108

Schneider, F. R. N., Sana, H., Evans, C. J., et al. 2018, Sci, 359, 69

Solomon, P. M., Rivolo, A. R., Barrett, J., \& Yahil, A. 1987, ApJ, 319, 730

Stodólkiewicz, J. S. 1963, AcA, 13, 30

Terlouw, J.-P., \& Vogelaar, M.-H.-R. 2015, Kapteyn Package version 2.3, http://www.astro.rug.nl/software/kapteyn/

Tilley, D. A., \& Pudritz, R. E. 2003, ApJ, 593, 426

Tokuda, K., Fukui, Y., Harada, R., et al. 2019, ApJ, 886, 15

van der Tak, F. F. S., Black, J. H., Schöier, F. L., Jansen, D. J., \& van Dishoeck, E. F. 2007, A\&A, 468, 627

Walborn, N. R., Barbá, R. H., \& Sewiło, M. M. 2013, AJ, 145, 98

Walborn, N. R., \& Blades, J. C. 1997, ApJS, 112, 457

Walsh, A. J., Breen, S. L., Britton, T., et al. 2011, MNRAS, 416, 1764

Werner, M. W., Becklin, E. E., Gatley, I., et al. 1978, MNRAS, 184, 365

Whitney, B. A., Sewilo, M., Indebetouw, R., et al. 2008, AJ, 136, 18

Williams, J. P., Blitz, L., \& Stark, A. A. 1995, ApJ, 451, 252

Williams, J. P., de Geus, E. J., \& Blitz, L. 2011, Clumpfind: Determining Structure in Molecular Clouds, Astrophysics Source Code Library, ascl:1107.014 